SECTION 4. Computer science, computer engineering and automation.

Alexandr Nikolayevich Shevtsov candidate of technical Sciences, President of International Academy International Academy of Theoretical \& Applied Sciences, (USA, Sweden, Kazakhstan) Shev_AlexXXXX@mail.ru

\title{
ADJUSTMENT LIBRARY WORD_TLB.DCU WHEN MIGRATING TO MICROSOFT OFFICE 2013
}

Abstract: The Work with the server actual Word for many software products. This article discusses the process of adjustment to the library Word_TLB.dcu to continue its use in the environment of the Word 2013.

Key words: server, text editor, test, program, system, code analysis, the description, block diagram, listing.

\section{КОРРЕКТИРОВКА БИБЛИОТЕКИ WОRD_TLВ.DCU ПРИ ПЕРЕХОДЕ НА MICROSOFT OFFICE 2013}

Аннотация: Работа с сервером Word актуальна для очень многих программных продуктов. В данной статье рассматривается процесс корректировки библиотеки Word_TLB.dси позволяюший продолжить ее использование в среде Word 2013.

Ключевые слова: сервер, текстовый редактор, тест, программа, система, код, анализ, описание, блок схема, листинг.

Процесс перехода на Microsoft Office 2013, для разработчика программного обеспечения реализующего работу с этим сервеером [1-6], сопровождается рядом неудобств и проблем (рис.1.).

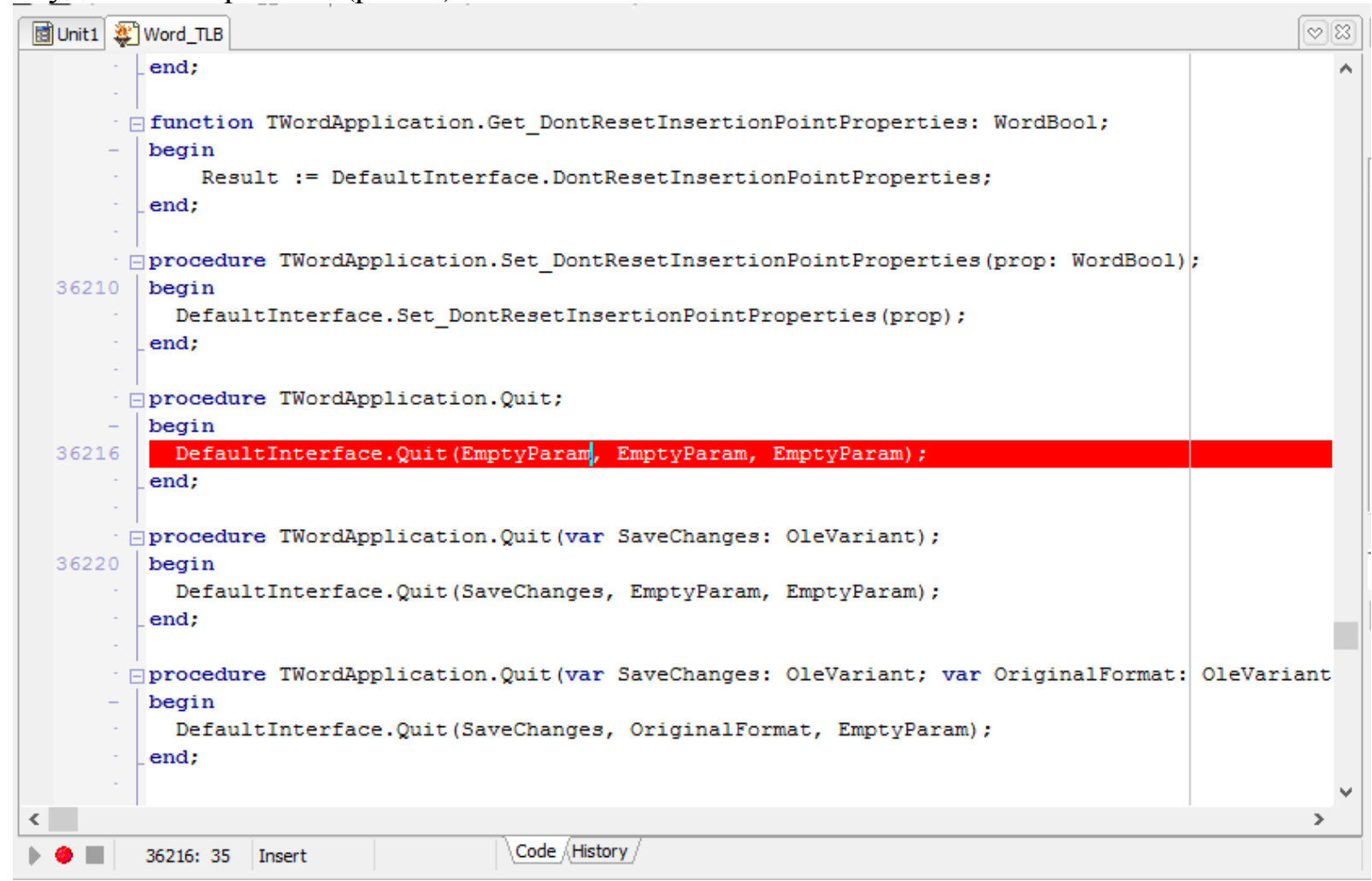

Рисунок 1 - Ошибка компиляции. 
Ошибка в работе наблюдается в этой процедуре:

procedure TWordApplication.Quit;

begin

DefaultInterface.Quit(EmptyParam, EmptyParam, EmptyParam);

end;

с данным кодом ошибки:

[dcc32 Error] Word_TLB.pas(36221): E2033 Types of actual and formal var parameters must be identical

Ошибка возникает по причине того, что используемая по умолчанию переменная «EmptyParam» не описана.

Опишем ее следующим образом:

procedure TWordApplication.Quit;

var

EmptyParam: OleVariant;

begin

DefaultInterface.Quit(EmptyParam, EmptyParam, EmptyParam); end;

Аналогичным образом внесем изменения также и в следующие процедуры:

- procedure TWordApplication.Quit(var SaveChanges: OleVariant);

- procedure TWordApplication.Quit(var SaveChanges: OleVariant; var OriginalFormat: OleVariant);

- procedure TWordApplication.PrintOutOld;

- procedure TWordApplication.PrintOutOld(var Background: OleVariant);

- procedure TWordApplication.PrintOutOld(var Background: OleVariant; var Append: OleVariant);

- procedure TWordApplication.PrintOutOld(var Background: OleVariant; var Append: OleVariant; var Range: OleVariant);

- procedure TWordApplication.PrintOutOld(var Background: OleVariant; var Append: OleVariant; var Range: OleVariant; var OutputFileName: OleVariant);

- procedure TWordApplication.PrintOutOld(var Background: OleVariant; var Append: OleVariant; var Range: OleVariant; var OutputFileName: OleVariant; var From: OleVariant);

- procedure TWordApplication.PrintOutOld(var Background: OleVariant; var Append: OleVariant; var Range: OleVariant; var OutputFileName: OleVariant; var From: OleVariant; var To_: OleVariant);

- procedure TWordApplication.PrintOutOld(var Background: OleVariant; var Append: OleVariant; var Range: OleVariant; var OutputFileName: OleVariant; var From: OleVariant; var To_: OleVariant; var Item: OleVariant);

- procedure TWordApplication.PrintOutOld(var Background: OleVariant; var Append: OleVariant; var Range: OleVariant; var OutputFileName: OleVariant; var From: OleVariant; var To_: OleVariant; var Item: OleVariant; var Copies: OleVariant); 
- procedure TWordApplication.PrintOutOld(var Background: OleVariant; var Append: OleVariant; var Range: OleVariant; var OutputFileName: OleVariant; var From: OleVariant; var To_: OleVariant; var Item: OleVariant; var Copies: OleVariant; var Pages: OleVariant);

- procedure TWordApplication.PrintOutOld(var Background: OleVariant; var Append: OleVariant; var Range: OleVariant; var OutputFileName: OleVariant; var From: OleVariant; var To_: OleVariant; var Item: OleVariant; var Copies: OleVariant; var Pages: OleVariant; var PageType: OleVariant);

- procedure TWordApplication.PrintOutOld(var Background: OleVariant; var Append: OleVariant; var Range: OleVariant; var OutputFileName: OleVariant; var From: OleVariant; var To_: OleVariant; var Item: OleVariant; var Copies: OleVariant; var Pages: OleVariant; var PageType: OleVariant; var PrintToFile: OleVariant);

- procedure TWordApplication.PrintOutOld(var Background: OleVariant; var Append: OleVariant; var Range: OleVariant; var OutputFileName: OleVariant;

- var From: OleVariant; var To_: OleVariant; var Item: OleVariant; var Copies: OleVariant; var Pages: OleVariant; var PageType: OleVariant; var PrintToFile: OleVariant; var Collate: OleVariant);

- procedure TWordApplication.PrintOutOld(var Background: OleVariant; var Append: OleVariant; var Range: OleVariant; var OutputFileName: OleVariant; var From: OleVariant; var To_: OleVariant; var Item: OleVariant; var Copies: OleVariant; var Pages: OleVariant; var PageType: OleVariant; var PrintToFile: OleVariant; var Collate: OleVariant; var FileName: OleVariant);

- procedure TWordApplication.PrintOutOld(var Background: OleVariant; var Append: OleVariant; var Range: OleVariant; var OutputFileName: OleVariant; var From: OleVariant; var To_: OleVariant; var Item: OleVariant; var Copies: OleVariant; var Pages: OleVariant; var PageType: OleVariant; var PrintToFile: OleVariant; var Collate: OleVariant; var FileName: OleVariant; var ActivePrinterMacGX: OleVariant);

- function TWordApplication.Repeat_: WordBool;

- function TWordApplication.Repeat_(var Times: OleVariant): WordBool;

- function TWordApplication.BuildKeyCode(Arg1: WdKey): Integer;

- function TWordApplication.BuildKeyCode(Arg1: WdKey; var Arg2: OleVariant): Integer;

- function TWordApplication.BuildKeyCode(Arg1: WdKey; var Arg2: OleVariant; var Arg3: OleVariant): Integer;

- function TWordApplication.KeyString(KeyCode: Integer): WideString;

- function TWordApplication.GetAddress: WideString;

- function TWordApplication.GetAddress(var Name: OleVariant): WideString;

- function TWordApplication.GetAddress(var Name: OleVariant; var AddressProperties: OleVariant): WideString;

- function TWordApplication.GetAddress(var Name: OleVariant; var AddressProperties: OleVariant; var UseAutoText: OleVariant): WideString;

- function TWordApplication.GetAddress(var Name: OleVariant; var AddressProperties: OleVariant; var UseAutoText: OleVariant; var DisplaySelectDialog: OleVariant): WideString;

- function TWordApplication.GetAddress(var Name: OleVariant; var AddressProperties: OleVariant; $\quad$ var UseAutoText: OleVariant; var DisplaySelectDialog: OleVariant; var SelectDialog: OleVariant): WideString;

- function TWordApplication.GetAddress(var Name: OleVariant; var AddressProperties: OleVariant; var UseAutoText: OleVariant; var DisplaySelectDialog: OleVariant; $\quad$ var SelectDialog: OleVariant; var CheckNamesDialog: OleVariant): WideString; 
- function TWordApplication.GetAddress(var Name: OleVariant; var AddressProperties: OleVariant; $\quad$ var UseAutoText: OleVariant; var DisplaySelectDialog: OleVariant; var SelectDialog: OleVariant; var CheckNamesDialog: OleVariant; var RecentAddressesChoice: OleVariant): WideString;

- function TWordApplication.CheckSpelling(const Word: WideString): WordBool;

- function TWordApplication.CheckSpelling(const Word: WideString; var CustomDictionary: OleVariant): WordBool;

- function TWordApplication.CheckSpelling(const Word: WideString; var CustomDictionary: OleVariant; var IgnoreUppercase: OleVariant): WordBool;

- function TWordApplication.CheckSpelling(const Word: WideString; var CustomDictionary: OleVariant; var IgnoreUppercase: OleVariant; var MainDictionary: OleVariant): WordBool;

- function TWordApplication.CheckSpelling(const Word: WideString; var CustomDictionary: OleVariant; var IgnoreUppercase: OleVariant; var MainDictionary: OleVariant; var CustomDictionary2: OleVariant): WordBool;

- function TWordApplication.CheckSpelling(const Word: WideString; var CustomDictionary: OleVariant; var IgnoreUppercase: OleVariant; var MainDictionary: OleVariant; var CustomDictionary2: OleVariant; var CustomDictionary3: OleVariant): WordBool;

- function TWordApplication.CheckSpelling(const Word: WideString; var CustomDictionary: OleVariant; var IgnoreUppercase: OleVariant; var MainDictionary: OleVariant; var CustomDictionary2: OleVariant; var CustomDictionary3: OleVariant; var CustomDictionary4: OleVariant): WordBool;

- function TWordApplication.CheckSpelling(const Word: WideString; var CustomDictionary: OleVariant; var IgnoreUppercase: OleVariant; var MainDictionary: OleVariant; var CustomDictionary2: OleVariant; var CustomDictionary3: OleVariant; var CustomDictionary4: OleVariant; var CustomDictionary5: OleVariant): WordBool;

- function TWordApplication.CheckSpelling(const Word: WideString; var CustomDictionary: OleVariant; var IgnoreUppercase: OleVariant; var MainDictionary: OleVariant; var CustomDictionary2: OleVariant; var CustomDictionary3: OleVariant; var CustomDictionary4: OleVariant; var CustomDictionary5: OleVariant; var CustomDictionary6: OleVariant): WordBool;

- function TWordApplication.CheckSpelling(const Word: WideString; var CustomDictionary: OleVariant; var IgnoreUppercase: OleVariant; var MainDictionary: OleVariant; var CustomDictionary2: OleVariant; var CustomDictionary3: OleVariant; var CustomDictionary4: OleVariant; var CustomDictionary5: OleVariant; var CustomDictionary6: OleVariant; var CustomDictionary7: OleVariant): WordBool;

- function TWordApplication.CheckSpelling(const Word: WideString; var CustomDictionary: OleVariant; var IgnoreUppercase: OleVariant; var MainDictionary: OleVariant; var CustomDictionary2: OleVariant; var CustomDictionary3: OleVariant; var CustomDictionary4: OleVariant; var CustomDictionary5: OleVariant; var CustomDictionary6: OleVariant; var CustomDictionary7: OleVariant; var CustomDictionary8: OleVariant): WordBool;

- function TWordApplication.CheckSpelling(const Word: WideString; var CustomDictionary: OleVariant; var IgnoreUppercase: OleVariant; var MainDictionary: OleVariant; var CustomDictionary2: OleVariant; var CustomDictionary3: OleVariant; var CustomDictionary4: OleVariant; var CustomDictionary5: OleVariant; var CustomDictionary6: OleVariant; var CustomDictionary7: OleVariant; var CustomDictionary8: OleVariant; var CustomDictionary9: OleVariant): WordBool; 
- function TWordApplication.GetSpellingSuggestions(const Word: WideString): SpellingSuggestions;

- function TWordApplication.GetSpellingSuggestions(const Word: WideString; var CustomDictionary: OleVariant): SpellingSuggestions;

- function TWordApplication.GetSpellingSuggestions(const Word: WideString; var CustomDictionary: OleVariant; var IgnoreUppercase: OleVariant): SpellingSuggestions;

- function TWordApplication.GetSpellingSuggestions(const Word: WideString; $\quad$ var CustomDictionary: OleVariant; var IgnoreUppercase: OleVariant; var MainDictionary: OleVariant): SpellingSuggestions;

- function TWordApplication.GetSpellingSuggestions(const Word: WideString; var CustomDictionary: OleVariant; var IgnoreUppercase: OleVariant; var MainDictionary: OleVariant; var SuggestionMode: OleVariant): SpellingSuggestions;

- function TWordApplication.GetSpellingSuggestions(const Word: WideString; var CustomDictionary: OleVariant; var IgnoreUppercase: OleVariant; $\quad$ var MainDictionary: OleVariant; var SuggestionMode: OleVariant; var CustomDictionary2: OleVariant): SpellingSuggestions;

- function TWordApplication.GetSpellingSuggestions(const Word: WideString; var CustomDictionary: OleVariant; var IgnoreUppercase: OleVariant; var MainDictionary: OleVariant; var SuggestionMode: OleVariant; var CustomDictionary2: OleVariant; var CustomDictionary3: OleVariant): SpellingSuggestions;

- function TWordApplication.GetSpellingSuggestions(const Word: WideString; var CustomDictionary: OleVariant; var IgnoreUppercase: OleVariant; var MainDictionary: OleVariant; var SuggestionMode: OleVariant; var CustomDictionary2: OleVariant; var CustomDictionary3: OleVariant; var CustomDictionary4: OleVariant): SpellingSuggestions;

- function TWordApplication.GetSpellingSuggestions(const Word: WideString; var CustomDictionary: OleVariant; var IgnoreUppercase: OleVariant; var MainDictionary: OleVariant; var SuggestionMode: OleVariant; var CustomDictionary2: OleVariant; var CustomDictionary3: OleVariant; var CustomDictionary4: OleVariant; var CustomDictionary5: OleVariant): SpellingSuggestions;

- function TWordApplication.GetSpellingSuggestions(const Word: WideString; var CustomDictionary: OleVariant; var IgnoreUppercase: OleVariant; var MainDictionary: OleVariant; var SuggestionMode: OleVariant; var CustomDictionary2: OleVariant; var CustomDictionary3: OleVariant; var CustomDictionary4: OleVariant; var CustomDictionary5: OleVariant; var CustomDictionary6: OleVariant): SpellingSuggestions;

- function TWordApplication.GetSpellingSuggestions(const Word: WideString; var CustomDictionary: OleVariant; var IgnoreUppercase: OleVariant; var MainDictionary: OleVariant; var SuggestionMode: OleVariant; var CustomDictionary2: OleVariant; var CustomDictionary3: OleVariant; var CustomDictionary4: OleVariant; var CustomDictionary5: OleVariant; var CustomDictionary6: OleVariant; var CustomDictionary7: OleVariant): SpellingSuggestions;

- function TWordApplication.GetSpellingSuggestions(const Word: WideString; var CustomDictionary: OleVariant; var IgnoreUppercase: OleVariant; var MainDictionary: OleVariant; var SuggestionMode: OleVariant; var CustomDictionary2: OleVariant; var CustomDictionary3: OleVariant; var CustomDictionary4: OleVariant; var CustomDictionary5: OleVariant; var CustomDictionary6: OleVariant; var 
CustomDictionary7: OleVariant; var CustomDictionary8: OleVariant): SpellingSuggestions;

- function TWordApplication.GetSpellingSuggestions(const Word: WideString; var CustomDictionary: OleVariant; var IgnoreUppercase: OleVariant; var MainDictionary: OleVariant; var SuggestionMode: OleVariant; var CustomDictionary2: OleVariant; var CustomDictionary3: OleVariant; var CustomDictionary4: OleVariant; var CustomDictionary5: OleVariant; var CustomDictionary6: OleVariant; var CustomDictionary7: OleVariant; var CustomDictionary8: OleVariant; var CustomDictionary9: OleVariant): SpellingSuggestions;

- procedure TWordApplication.OnTime(var When: OleVariant; const Name: WideString);

- function TWordApplication.MountVolume(const Zone: WideString; const Server: WideString;const Volume: WideString): Smallint;

- function TWordApplication.MountVolume(const Zone: WideString; const Server: WideString; const Volume: WideString; var User: OleVariant): Smallint;

- function TWordApplication.MountVolume(const Zone: WideString; const Server: WideString; const Volume: WideString; var User: OleVariant;var UserPassword: OleVariant): Smallint;

- function TWordApplication.PointsToPixels(Points: Single): Single;

- function TWordApplication.PixelsToPoints(Pixels: Single): Single;

- procedure TWordApplication.PrintOut2000;

- procedure TWordApplication.PrintOut2000(var Background: OleVariant);

- procedure TWordApplication.PrintOut2000(var Background: OleVariant; var Append: OleVariant);

- procedure TWordApplication.PrintOut2000(var Background: OleVariant; var Append: OleVariant; var Range: OleVariant);

- procedure TWordApplication.PrintOut2000(var Background: OleVariant; var Append: OleVariant; var Range: OleVariant; var OutputFileName: OleVariant);

- procedure TWordApplication.PrintOut2000(var Background: OleVariant; var Append: OleVariant; var Range: OleVariant; var OutputFileName: OleVariant; var From: OleVariant);

- procedure TWordApplication.PrintOut2000(var Background: OleVariant; var Append: OleVariant; var Range: OleVariant; var OutputFileName: OleVariant; var From: OleVariant; var To_: OleVariant);

- procedure TWordApplication.PrintOut2000(var Background: OleVariant; var Append: OleVariant; var Range: OleVariant; var OutputFileName: OleVariant; var From: OleVariant; var To_: OleVariant; var Item: OleVariant);

- procedure TWordApplication.PrintOut2000(var Background: OleVariant; var Append: OleVariant; var Range: OleVariant; var OutputFileName: OleVariant; var From: OleVariant; var To_: OleVariant; var Item: OleVariant; var Copies: OleVariant);

- procedure TWordApplication.PrintOut2000(var Background: OleVariant; var Append: OleVariant; var Range: OleVariant; var OutputFileName: OleVariant; var From: OleVariant; var To_: OleVariant; var Item: OleVariant; var Copies: OleVariant; var Pages: OleVariant);

- procedure TWordApplication.PrintOut2000(var Background: OleVariant; var Append: OleVariant; var Range: OleVariant; var OutputFileName: OleVariant; var From: OleVariant; var To_: OleVariant; var Item: OleVariant; var Copies: OleVariant; var Pages: OleVariant; var PageType: OleVariant);

- procedure TWordApplication.PrintOut2000(var Background: OleVariant; var Append: OleVariant; var Range: OleVariant; var OutputFileName: OleVariant; var From: 
OleVariant; var To_: OleVariant; var Item: OleVariant; var Copies: OleVariant; var Pages: OleVariant; var PageType: OleVariant; var PrintToFile: OleVariant);

- procedure TWordApplication.PrintOut2000(var Background: OleVariant; var Append: OleVariant; var Range: OleVariant; var OutputFileName: OleVariant; var From: OleVariant; var To_: OleVariant; var Item: OleVariant; var Copies: OleVariant; var Pages: OleVariant; var PageType: OleVariant; var PrintToFile: OleVariant; var Collate: OleVariant);

- procedure TWordApplication.PrintOut2000(var Background: OleVariant; var Append: OleVariant; var Range: OleVariant; var OutputFileName: OleVariant; var From: OleVariant; var To_: OleVariant; var Item: OleVariant; var Copies: OleVariant; var Pages: OleVariant; var PageType: OleVariant; var PrintToFile: OleVariant; var Collate: OleVariant; var FileName: OleVariant);

- procedure TWordApplication.PrintOut2000(var Background: OleVariant; var Append: OleVariant; var Range: OleVariant; var OutputFileName: OleVariant; var From: OleVariant; var To_: OleVariant; var Item: OleVariant; var Copies: OleVariant; var Pages: OleVariant; var PageType: OleVariant; var PrintToFile: OleVariant; var Collate: OleVariant; var FileName: OleVariant; var ActivePrinterMacGX: OleVariant);

- procedure TWordApplication.PrintOut2000(var Background: OleVariant; var Append: OleVariant; var Range: OleVariant; var OutputFileName: OleVariant; var From: OleVariant; var To_: OleVariant; var Item: OleVariant; var Copies: OleVariant; var Pages: OleVariant; var PageType: OleVariant; var PrintToFile: OleVariant; var Collate: OleVariant; var FileName: OleVariant; var ActivePrinterMacGX: OleVariant; var ManualDuplexPrint: OleVariant);

- procedure TWordApplication.PrintOut2000(var Background: OleVariant; var Append: OleVariant; var Range: OleVariant; var OutputFileName: OleVariant; var From: OleVariant; var To_: OleVariant; var Item: OleVariant; var Copies: OleVariant; var Pages: OleVariant; var PageType: OleVariant; var PrintToFile: OleVariant; var Collate: OleVariant; var FileName: OleVariant; var ActivePrinterMacGX: OleVariant; var ManualDuplexPrint: OleVariant; var PrintZoomColumn: OleVariant);

- procedure TWordApplication.PrintOut2000(var Background: OleVariant; var Append: OleVariant; var Range: OleVariant; var OutputFileName: OleVariant; var From: OleVariant; var To_: OleVariant; var Item: OleVariant; var Copies: OleVariant; var Pages: OleVariant; var PageType: OleVariant; var PrintToFile: OleVariant; var Collate: OleVariant; var FileName: OleVariant; var ActivePrinterMacGX: OleVariant; var ManualDuplexPrint: OleVariant; var PrintZoomColumn: OleVariant; var PrintZoomRow: OleVariant);

- procedure TWordApplication.PrintOut2000(var Background: OleVariant; var Append: OleVariant; var Range: OleVariant; var OutputFileName: OleVariant; var From: OleVariant; var To_: OleVariant; var Item: OleVariant; var Copies: OleVariant; var Pages: OleVariant; var PageType: OleVariant; var PrintToFile: OleVariant; var Collate: OleVariant; var FileName: OleVariant; var ActivePrinterMacGX: OleVariant; var ManualDuplexPrint: OleVariant; var PrintZoomColumn: OleVariant; var PrintZoomRow: OleVariant; var PrintZoomPaperWidth: OleVariant);

- function TWordApplication.Run(const MacroName: WideString): OleVariant;

- function TWordApplication.Run(const MacroName: WideString; var varg1: OleVariant): OleVariant;

- function TWordApplication.Run(const MacroName: WideString; var varg1: OleVariant; var varg2: OleVariant): OleVariant;

- function TWordApplication.Run(const MacroName: WideString; var varg1: OleVariant; var varg2: OleVariant; var varg3: OleVariant): OleVariant; 
- function TWordApplication.Run(const MacroName: WideString; var varg1: OleVariant; var varg2: OleVariant; var varg3: OleVariant; var varg4: OleVariant): OleVariant;

- function TWordApplication.Run(const MacroName: WideString; var varg1: OleVariant; var varg2: OleVariant; var varg3: OleVariant; var varg4: OleVariant; var varg5: OleVariant): OleVariant;

- function TWordApplication.Run(const MacroName: WideString; var varg1: OleVariant; var varg2: OleVariant; var varg3: OleVariant; var varg4: OleVariant; var varg5: OleVariant; var varg6: OleVariant): OleVariant;

- function TWordApplication.Run(const MacroName: WideString; var varg1: OleVariant; var varg2: OleVariant; var varg3: OleVariant; var varg4: OleVariant; var varg5: OleVariant; var varg6: OleVariant; var varg7: OleVariant; var varg8: OleVariant): OleVariant;

- function TWordApplication.Run(const MacroName: WideString; var varg1: OleVariant; var varg2: OleVariant; var varg3: OleVariant; var varg4: OleVariant; var varg5: OleVariant; var varg6: OleVariant; var varg7: OleVariant; var varg8: OleVariant; var varg9: OleVariant): OleVariant;

- function TWordApplication.Run(const MacroName: WideString; var varg1: OleVariant; var varg2: OleVariant; var varg3: OleVariant; var varg4: OleVariant; var varg5: OleVariant; var varg6: OleVariant; var varg7: OleVariant; var varg8: OleVariant; var varg9: OleVariant; var varg10: OleVariant): OleVariant;

- function TWordApplication.Run(const MacroName: WideString; var varg1: OleVariant; var varg2: OleVariant; var varg3: OleVariant; var varg4: OleVariant; var varg5: OleVariant; var varg6: OleVariant; var varg7: OleVariant; var varg8: OleVariant; var varg9: OleVariant; var varg10: OleVariant; var varg11: OleVariant): OleVariant;

- function TWordApplication.Run(const MacroName: WideString; var varg1: OleVariant; var varg2: OleVariant; var varg3: OleVariant; var varg4: OleVariant; var varg5: OleVariant; var varg6: OleVariant; var varg7: OleVariant; var varg8: OleVariant; var varg9: OleVariant; var varg10: OleVariant; var varg11: OleVariant; var varg12: OleVariant): OleVariant;

- function TWordApplication.Run(const MacroName: WideString; var varg1: OleVariant; var varg2: OleVariant; var varg3: OleVariant; var varg4: OleVariant; var varg5: OleVariant; var varg6: OleVariant; var varg7: OleVariant; var varg8: OleVariant; var varg9: OleVariant; var varg10: OleVariant; var varg11: OleVariant; var varg12: OleVariant; var varg13: OleVariant): OleVariant;

- function TWordApplication.Run(const MacroName: WideString; var varg1: OleVariant; var varg2: OleVariant; var varg3: OleVariant; var varg4: OleVariant; var varg5: OleVariant; var varg6: OleVariant; var varg7: OleVariant; var varg8: OleVariant; var varg9: OleVariant; var varg10: OleVariant; var varg11: OleVariant; var varg12: OleVariant; var varg13: OleVariant; var varg14: OleVariant): OleVariant;

- function TWordApplication.Run(const MacroName: WideString; var varg1: OleVariant; var varg2: OleVariant; var varg3: OleVariant; var varg4: OleVariant; var varg5: OleVariant; var varg6: OleVariant; var varg7: OleVariant; var varg8: OleVariant; var varg9: OleVariant; var varg10: OleVariant; var varg11: OleVariant; var varg12: OleVariant; var varg13: OleVariant; var varg14: OleVariant; var varg15: OleVariant): OleVariant;

- function TWordApplication.Run(const MacroName: WideString; var varg1: OleVariant; var varg2: OleVariant; var varg3: OleVariant; var varg4: OleVariant; var varg5: OleVariant; var varg6: OleVariant; var varg7: OleVariant; var varg8: OleVariant; var varg9: OleVariant; var varg10: OleVariant; var varg11: OleVariant; var varg12: 
OleVariant; var varg13: OleVariant; var varg14: OleVariant; var varg15: OleVariant; var varg16: OleVariant): OleVariant;

- function TWordApplication.Run(const MacroName: WideString; var varg1: OleVariant; var varg2: OleVariant; var varg3: OleVariant; var varg4: OleVariant; var varg5: OleVariant; var varg6: OleVariant; var varg7: OleVariant; var varg8: OleVariant; var varg9: OleVariant; var varg10: OleVariant; var varg11: OleVariant; var varg12: OleVariant; var varg13: OleVariant; var varg14: OleVariant; var varg15: OleVariant; var varg16: OleVariant; var varg17: OleVariant): OleVariant;

- function TWordApplication.Run(const MacroName: WideString; var varg1: OleVariant; var varg2: OleVariant; var varg3: OleVariant; var varg4: OleVariant; var varg5: OleVariant; var varg6: OleVariant; var varg7: OleVariant; var varg8: OleVariant; var varg9: OleVariant; var varg10: OleVariant; var varg11: OleVariant; var varg12: OleVariant; var varg13: OleVariant; var varg14: OleVariant; var varg15: OleVariant; var varg16: OleVariant; var varg17: OleVariant; var varg18: OleVariant): OleVariant;

- function TWordApplication.Run(const MacroName: WideString; var varg1: OleVariant; var varg2: OleVariant; var varg3: OleVariant; var varg4: OleVariant; var varg5: OleVariant; var varg6: OleVariant; var varg7: OleVariant; var varg8: OleVariant; var varg9: OleVariant; var varg10: OleVariant; var varg11: OleVariant; var varg12: OleVariant; var varg13: OleVariant; var varg14: OleVariant; var varg15: OleVariant; var varg16: OleVariant; var varg17: OleVariant; var varg18: OleVariant; var varg19: OleVariant): OleVariant;

- function TWordApplication.Run(const MacroName: WideString; var varg1: OleVariant; var varg2: OleVariant; var varg3: OleVariant; var varg4: OleVariant; var varg5: OleVariant; var varg6: OleVariant; var varg7: OleVariant; var varg8: OleVariant; var varg9: OleVariant; var varg10: OleVariant; var varg11: OleVariant; var varg12: OleVariant; var varg13: OleVariant; var varg14: OleVariant; var varg15: OleVariant; var varg16: OleVariant; var varg17: OleVariant; var varg18: OleVariant; var varg19: OleVariant; var varg20: OleVariant): OleVariant;

- function TWordApplication.Run(const MacroName: WideString; var varg1: OleVariant; var varg2: OleVariant; var varg3: OleVariant; var varg4: OleVariant; var varg5: OleVariant; var varg6: OleVariant; var varg7: OleVariant; var varg8: OleVariant; var varg9: OleVariant; var varg10: OleVariant; var varg11: OleVariant; var varg12: OleVariant; var varg13: OleVariant; var varg14: OleVariant; var varg15: OleVariant; var varg16: OleVariant; var varg17: OleVariant; var varg18: OleVariant; var varg19: OleVariant; var varg20: OleVariant; var varg21: OleVariant): OleVariant;

- function TWordApplication.Run(const MacroName: WideString; var varg1: OleVariant; var varg2: OleVariant; var varg3: OleVariant; var varg4: OleVariant; var varg5: OleVariant; var varg6: OleVariant; var varg7: OleVariant; var varg8: OleVariant; var varg9: OleVariant; var varg10: OleVariant; var varg11: OleVariant; var varg12: OleVariant; var varg13: OleVariant; var varg14: OleVariant; var varg15: OleVariant; var varg16: OleVariant; var varg17: OleVariant; var varg18: OleVariant; var varg19: OleVariant; var varg20: OleVariant; var varg21: OleVariant; var varg22: OleVariant): OleVariant;

- function TWordApplication.Run(const MacroName: WideString; var varg1: OleVariant; var varg2: OleVariant; var varg3: OleVariant; var varg4: OleVariant; var varg5: OleVariant; var varg6: OleVariant; var varg7: OleVariant; var varg8: OleVariant; var varg9: OleVariant; var varg10: OleVariant; var varg11: OleVariant; var varg12: OleVariant; var varg13: OleVariant; var varg14: OleVariant; var varg15: OleVariant; var varg16: OleVariant; var varg17: OleVariant; var varg18: OleVariant; var varg19: OleVariant; var varg20: OleVariant; var varg21: OleVariant; var varg22: OleVariant; var varg23: OleVariant): OleVariant; 
- function TWordApplication.Run(const MacroName: WideString; var varg1: OleVariant; var varg2: OleVariant; var varg3: OleVariant; var varg4: OleVariant; var varg5: OleVariant; var varg6: OleVariant; var varg7: OleVariant; var varg8: OleVariant; var varg9: OleVariant; var varg10: OleVariant; var varg11: OleVariant; var varg12: OleVariant; var varg13: OleVariant; var varg14: OleVariant; var varg15: OleVariant; var varg16: OleVariant; var varg17: OleVariant; var varg18: OleVariant; var varg19: OleVariant; var varg20: OleVariant; var varg21: OleVariant; var varg22: OleVariant; var varg23: OleVariant; var varg24: OleVariant): OleVariant;

- function TWordApplication.Run(const MacroName: WideString; var varg1: OleVariant; var varg2: OleVariant; var varg3: OleVariant; var varg4: OleVariant; var varg5: OleVariant; var varg6: OleVariant; var varg7: OleVariant; var varg8: OleVariant; var varg9: OleVariant; var varg10: OleVariant; var varg11: OleVariant; var varg12: OleVariant; var varg13: OleVariant; var varg14: OleVariant; var varg15: OleVariant; var varg16: OleVariant; var varg17: OleVariant; var varg18: OleVariant; var varg19: OleVariant; var varg20: OleVariant; var varg21: OleVariant; var varg22: OleVariant; var varg23: OleVariant; var varg24: OleVariant; var varg25: OleVariant): OleVariant;

- function TWordApplication.Run(const MacroName: WideString; var varg1: OleVariant; var varg2: OleVariant; var varg3: OleVariant; var varg4: OleVariant; var varg5: OleVariant; var varg6: OleVariant; var varg7: OleVariant; var varg8: OleVariant; var varg9: OleVariant; var varg10: OleVariant; var varg11: OleVariant; var varg12: OleVariant; var varg13: OleVariant; var varg14: OleVariant; var varg15: OleVariant; var varg16: OleVariant; var varg17: OleVariant; var varg18: OleVariant; var varg19: OleVariant; var varg20: OleVariant; var varg21: OleVariant; var varg22: OleVariant; var varg23: OleVariant; var varg24: OleVariant; var varg25: OleVariant; var varg26: OleVariant): OleVariant;

- function TWordApplication.Run(const MacroName: WideString; var varg1: OleVariant; var varg2: OleVariant; var varg3: OleVariant; var varg4: OleVariant; var varg5: OleVariant; var varg6: OleVariant; var varg7: OleVariant; var varg8: OleVariant; var varg9: OleVariant; var varg10: OleVariant; var varg11: OleVariant; var varg12: OleVariant; var varg13: OleVariant; var varg14: OleVariant; var varg15: OleVariant; var varg16: OleVariant; var varg17: OleVariant; var varg18: OleVariant; var varg19: OleVariant; var varg20: OleVariant; var varg21: OleVariant; var varg22: OleVariant; var varg23: OleVariant; var varg24: OleVariant; var varg25: OleVariant; var varg26: OleVariant; var varg27: OleVariant): OleVariant;

- function TWordApplication.Run(const MacroName: WideString; var varg1: OleVariant; var varg2: OleVariant; var varg3: OleVariant; var varg4: OleVariant; var varg5: OleVariant; var varg6: OleVariant; var varg7: OleVariant; var varg8: OleVariant; var varg9: OleVariant; var varg10: OleVariant; var varg11: OleVariant; var varg12: OleVariant; var varg13: OleVariant; var varg14: OleVariant; var varg15: OleVariant; var varg16: OleVariant; var varg17: OleVariant; var varg18: OleVariant; var varg19: OleVariant; var varg20: OleVariant; var varg21: OleVariant; var varg22: OleVariant; var varg23: OleVariant; var varg24: OleVariant; var varg25: OleVariant; var varg26: OleVariant; var varg27: OleVariant; var varg28: OleVariant): OleVariant;

- function TWordApplication.Run(const MacroName: WideString; var varg1: OleVariant; var varg2: OleVariant; var varg3: OleVariant; var varg4: OleVariant; var varg5: OleVariant; var varg6: OleVariant; var varg7: OleVariant; var varg8: OleVariant; var varg9: OleVariant; var varg10: OleVariant; var varg11: OleVariant; var varg12: OleVariant; var varg13: OleVariant; var varg14: OleVariant; var varg15: OleVariant; var varg16: OleVariant; var varg17: OleVariant; var varg18: OleVariant; var varg19: OleVariant; var varg20: OleVariant; var varg21: OleVariant; var varg22: OleVariant; var varg23: OleVariant; var varg24: OleVariant; var varg25: OleVariant; var varg26: 
OleVariant; var varg27: OleVariant; var varg28: OleVariant; var varg29: OleVariant): OleVariant;

- procedure TWordApplication.PrintOut;

- procedure TWordApplication.PrintOut(var Background: OleVariant);

- procedure TWordApplication.PrintOut(var Background: OleVariant; var Append: OleVariant);

- procedure TWordApplication.PrintOut(var Background: OleVariant; var Append: OleVariant; $\quad$ var Range: OleVariant);

- procedure TWordApplication.PrintOut(var Background: OleVariant; var Append: OleVariant; $\quad$ var Range: OleVariant; var OutputFileName: OleVariant);

- procedure TWordApplication.PrintOut(var Background: OleVariant; var Append: OleVariant; var Range: OleVariant; var OutputFileName: OleVariant; $\quad$ var From: OleVariant);

- procedure TWordApplication.PrintOut(var Background: OleVariant; var Append: OleVariant; $\quad$ var Range: OleVariant; var OutputFileName: OleVariant; $\quad$ var From: OleVariant; var To_: OleVariant);

- procedure TWordApplication.PrintOut(var Background: OleVariant; var Append: OleVariant; $\quad$ var Range: OleVariant; var OutputFileName: OleVariant; $\quad$ var From: OleVariant; var To_: OleVariant; var Item: OleVariant);

- procedure TWordApplication.PrintOut(var Background: OleVariant; var Append: OleVariant; var Range: OleVariant; var OutputFileName: OleVariant; var From: OleVariant; var To_: OleVariant; $\quad$ var Item: OleVariant; var Copies: OleVariant);

- procedure TWordApplication.PrintOut(var Background: OleVariant; var Append: OleVariant; $\quad$ var Range: OleVariant; var OutputFileName: OleVariant; var From: OleVariant; var To_: OleVariant; $\quad$ var Item: OleVariant; var Copies: OleVariant; var Pages: OleVariant);

- procedure TWordApplication.PrintOut(var Background: OleVariant; var Append: OleVariant; $\quad$ var Range: OleVariant; var OutputFileName: OleVariant; $\quad$ var From: OleVariant; var To_: OleVariant; var Item: OleVariant; var Copies: OleVariant; var Pages: OleVariant; var PageType: OleVariant);

- procedure TWordApplication.PrintOut(var Background: OleVariant; var Append: OleVariant; var Range: OleVariant; var OutputFileName: OleVariant; var From: OleVariant; var To_: OleVariant; var Item: OleVariant; var Copies: OleVariant; var Pages: OleVariant; var PageType: OleVariant; $\quad$ var PrintToFile: OleVariant);

- procedure TWordApplication.PrintOut(var Background: OleVariant; var Append: OleVariant; var Range: OleVariant; var OutputFileName: OleVariant; var From: OleVariant; var To_: OleVariant; var Item: OleVariant; var Copies: OleVariant; var Pages: OleVariant; var PageType: OleVariant; $\quad$ var PrintToFile: OleVariant; var Collate: OleVariant);

- procedure TWordApplication.PrintOut(var Background: OleVariant; var Append: OleVariant; $\quad$ var Range: OleVariant; var OutputFileName: OleVariant; $\quad$ var From: OleVariant; var To_: OleVariant; var Item: OleVariant; var Copies: OleVariant; var Pages: OleVariant; var PageType: OleVariant; $\quad$ var PrintToFile: OleVariant; var Collate: OleVariant; $\quad$ var FileName: OleVariant);

- procedure TWordApplication.PrintOut(var Background: OleVariant; var Append: OleVariant; $\quad$ var Range: OleVariant; var OutputFileName: OleVariant; $\quad$ var From: OleVariant; var To_: OleVariant; var Item: OleVariant; var Copies: OleVariant; var Pages: OleVariant; var PageType: OleVariant; $\quad$ var PrintToFile: OleVariant; var Collate: OleVariant; var FileName: OleVariant; var ActivePrinterMacGX: OleVariant); 
- procedure TWordApplication.PrintOut(var Background: OleVariant; var Append: OleVariant; var Range: OleVariant; var OutputFileName: OleVariant; var From: OleVariant; var To_: OleVariant; $\quad$ var Item: OleVariant; var Copies: OleVariant; var Pages: OleVariant; var PageType: OleVariant; var PrintToFile: OleVariant; var Collate: OleVariant; $\quad$ var FileName: OleVariant; var ActivePrinterMacGX: OleVariant; var ManualDuplexPrint: OleVariant);

- procedure TWordApplication.PrintOut(var Background: OleVariant; var Append: OleVariant; $\quad$ var Range: OleVariant; var OutputFileName: OleVariant; $\quad$ var From: OleVariant; var To_: OleVariant; var Item: OleVariant; var Copies: OleVariant; var Pages: OleVariant; var PageType: OleVariant; $\quad$ var PrintToFile: OleVariant; var Collate: OleVariant; $\quad$ var FileName: OleVariant; var ActivePrinterMacGX: OleVariant; var ManualDuplexPrint: OleVariant; var PrintZoomColumn: OleVariant);procedure TWordApplication.PrintOut(var Background: OleVariant; var Append: OleVariant; var Range: OleVariant; var OutputFileName: OleVariant; var From: OleVariant; var To_: OleVariant; var Item: OleVariant; var Copies: OleVariant; var Pages: OleVariant; var PageType: OleVariant; var PrintToFile: OleVariant; var Collate: OleVariant; $\quad$ var FileName: OleVariant; var ActivePrinterMacGX: OleVariant; var ManualDuplexPrint: OleVariant; var PrintZoomColumn: OleVariant; var PrintZoomRow: OleVariant);

- procedure TWordApplication.PrintOut(var Background: OleVariant; var Append: OleVariant; var Range: OleVariant; var OutputFileName: OleVariant; var From: OleVariant; var To_: OleVariant; var Item: OleVariant; var Copies: OleVariant; var Pages: OleVariant; var PageType: OleVariant; $\quad$ var PrintToFile: OleVariant; var Collate: OleVariant; $\quad$ var FileName: OleVariant; var ActivePrinterMacGX: OleVariant; var ManualDuplexPrint: OleVariant; var PrintZoomColumn: OleVariant; var PrintZoomRow: OleVariant; $\quad$ var PrintZoomPaperWidth: OleVariant);

- procedure TWordDocument.Close;

- procedure TWordDocument.Close(var SaveChanges: OleVariant);

- procedure TWordDocument.Close(var SaveChanges: OleVariant; var OriginalFormat: OleVariant);

- procedure TWordDocument.SaveAs2000;

- procedure TWordDocument.SaveAs2000(var FileName: OleVariant);

- procedure TWordDocument.SaveAs2000(var FileName: OleVariant; var FileFormat: OleVariant);

- procedure TWordDocument.SaveAs2000(var FileName: OleVariant; var FileFormat: OleVariant; $\quad$ var LockComments: OleVariant);

- procedure TWordDocument.SaveAs2000(var FileName: OleVariant; var FileFormat: OleVariant; $\quad$ var LockComments: OleVariant; var Password: OleVariant);

- procedure TWordDocument.SaveAs2000(var FileName: OleVariant; var FileFormat: OleVariant; var LockComments: OleVariant; var Password: OleVariant; var AddToRecentFiles: OleVariant);

- procedure TWordDocument.SaveAs2000(var FileName: OleVariant; var FileFormat: OleVariant; var LockComments: OleVariant; var Password: OleVariant; var AddToRecentFiles: OleVariant; var WritePassword: OleVariant);

- procedure TWordDocument.SaveAs2000(var FileName: OleVariant; var FileFormat: OleVariant; var LockComments: OleVariant; var Password: OleVariant; var AddToRecentFiles: OleVariant; var WritePassword: OleVariant; var ReadOnlyRecommended: OleVariant);

- procedure TWordDocument.SaveAs2000(var FileName: OleVariant; var FileFormat: OleVariant; $\quad$ var LockComments: OleVariant; var Password: OleVariant; var 
AddToRecentFiles: OleVariant; var WritePassword: OleVariant; var ReadOnlyRecommended: OleVariant; var EmbedTrueTypeFonts: OleVariant);

- procedure TWordDocument.SaveAs2000(var FileName: OleVariant; var FileFormat: OleVariant; var LockComments: OleVariant; var Password: OleVariant; var AddToRecentFiles: OleVariant; var WritePassword: OleVariant; var ReadOnlyRecommended: OleVariant; var EmbedTrueTypeFonts: OleVariant; var SaveNativePictureFormat: OleVariant);

- procedure TWordDocument.SaveAs2000(var FileName: OleVariant; var FileFormat: OleVariant; var LockComments: OleVariant; var Password: OleVariant; var AddToRecentFiles: OleVariant; var WritePassword: OleVariant; var ReadOnlyRecommended: OleVariant; var EmbedTrueTypeFonts: OleVariant; var SaveNativePictureFormat: OleVariant; $\quad$ var SaveFormsData: OleVariant); procedure TWordDocument.PrintOutOld;

- procedure TWordDocument.PrintOutOld(var Background: OleVariant);

- procedure TWordDocument.PrintOutOld(var Background: OleVariant; var Append: OleVariant);

- procedure TWordDocument.PrintOutOld(var Background: OleVariant; var Append: OleVariant; $\quad$ var Range: OleVariant);

- procedure TWordDocument.PrintOutOld(var Background: OleVariant; var Append: OleVariant; $\quad$ var Range: OleVariant; var OutputFileName: OleVariant);

- procedure TWordDocument.PrintOutOld(var Background: OleVariant; var Append: OleVariant; var Range: OleVariant; var OutputFileName: OleVariant; var From: OleVariant);

- procedure TWordDocument.PrintOutOld(var Background: OleVariant; var Append: OleVariant; $\quad$ var Range: OleVariant; var OutputFileName: OleVariant; $\quad$ var From: OleVariant; var To_: OleVariant);

- procedure TWordDocument.PrintOutOld(var Background: OleVariant; var Append: OleVariant; $\quad$ var Range: OleVariant; var OutputFileName: OleVariant; $\quad$ var From: OleVariant; var To_: OleVariant; var Item: OleVariant);

- procedure TWordDocument.PrintOutOld(var Background: OleVariant; var Append: OleVariant; $\quad$ var Range: OleVariant; var OutputFileName: OleVariant; $\quad$ var From: OleVariant; var To_: OleVariant; $\quad$ var Item: OleVariant; var Copies: OleVariant);

- procedure TWordDocument.PrintOutOld(var Background: OleVariant; var Append: OleVariant; var Range: OleVariant; var OutputFileName: OleVariant; var From: OleVariant; var To_: OleVariant; $\quad$ var Item: OleVariant; var Copies: OleVariant; var Pages: OleVariant);

- procedure TWordDocument.PrintOutOld(var Background: OleVariant; var Append: OleVariant; $\quad$ var Range: OleVariant; var OutputFileName: OleVariant; var From: OleVariant; var To_: OleVariant; var Item: OleVariant; var Copies: OleVariant; var Pages: OleVariant; var PageType: OleVariant);

- procedure TWordDocument.PrintOutOld(var Background: OleVariant; var Append: OleVariant; $\quad$ var Range: OleVariant; var OutputFileName: OleVariant; $\quad$ var From: OleVariant; var To_: OleVariant; var Item: OleVariant; var Copies: OleVariant; var Pages: OleVariant; var PageType: OleVariant; $\quad$ var PrintToFile: OleVariant);

- procedure TWordDocument.PrintOutOld(var Background: OleVariant; var Append: OleVariant; $\quad$ var Range: OleVariant; var OutputFileName: OleVariant; $\quad$ var From: OleVariant; var To_: OleVariant; var Item: OleVariant; var Copies: OleVariant; var Pages: OleVariant; var PageType: OleVariant; $\quad$ var PrintToFile: OleVariant; var Collate: OleVariant); 
- procedure TWordDocument.PrintOutOld(var Background: OleVariant; var Append: OleVariant; var Range: OleVariant; var OutputFileName: OleVariant; var From: OleVariant; var To_: OleVariant; $\quad$ var Item: OleVariant; var Copies: OleVariant; var Pages: OleVariant; var PageType: OleVariant; var PrintToFile: OleVariant; var Collate: OleVariant; $\quad$ var ActivePrinterMacGX: OleVariant);

- function TWordDocument.Range: WordRange;

- function TWordDocument.Range(var Start: OleVariant): WordRange;

- function TWordDocument.GoTo_: WordRange;

- function TWordDocument.GoTo_(var What: OleVariant): WordRange;

- function TWordDocument.GoTo_(var What: OleVariant; var Which: OleVariant): WordRange;

- function TWordDocument.GoTo_(var What: OleVariant; var Which: OleVariant; var Count: OleVariant): WordRange;

- function TWordDocument.Undo: WordBool;

- function TWordDocument.Redo: WordBool;

- function TWordDocument.ComputeStatistics(Statistic: WdStatistic): Integer;

- procedure TWordDocument.Protect2002(Type_: WdProtectionType);

- procedure TWordDocument.Protect2002(Type_: WdProtectionType; var NoReset: OleVariant);

- procedure TWordDocument.Unprotect;

- procedure TWordDocument.EditionOptions(Type_: WdEditionType; Option: WdEditionOption; const Name: WideString);

- procedure TWordDocument.RunLetterWizard;

- procedure TWordDocument.CheckSpelling;

- procedure TWordDocument.CheckSpelling(var CustomDictionary: OleVariant);

- procedure TWordDocument.CheckSpelling(var CustomDictionary: OleVariant;var IgnoreUppercase: OleVariant);

- procedure TWordDocument.CheckSpelling(var CustomDictionary: OleVariant;var IgnoreUppercase: OleVariant; var AlwaysSuggest: OleVariant);

- procedure TWordDocument.CheckSpelling(var CustomDictionary: OleVariant;var IgnoreUppercase: OleVariant;var AlwaysSuggest: OleVariant;var CustomDictionary2: OleVariant);

- procedure TWordDocument.CheckSpelling(var CustomDictionary: OleVariant;var IgnoreUppercase: OleVariant;var AlwaysSuggest: OleVariant;var CustomDictionary2: OleVariant;var CustomDictionary3: OleVariant);

- procedure TWordDocument.CheckSpelling(var CustomDictionary: OleVariant;var IgnoreUppercase: OleVariant;var AlwaysSuggest: OleVariant;var CustomDictionary2: OleVariant;var CustomDictionary3: OleVariant;var CustomDictionary4: OleVariant);

- procedure TWordDocument.CheckSpelling(var CustomDictionary: OleVariant;var IgnoreUppercase: OleVariant;var AlwaysSuggest: OleVariant;var CustomDictionary2: OleVariant;var CustomDictionary3: OleVariant;var CustomDictionary4: OleVariant;var CustomDictionary5: OleVariant);

- procedure TWordDocument.CheckSpelling(var CustomDictionary: OleVariant;var IgnoreUppercase: OleVariant;var AlwaysSuggest: OleVariant;var CustomDictionary2: OleVariant;var CustomDictionary3: OleVariant;var CustomDictionary4: OleVariant;var CustomDictionary5: OleVariant;var CustomDictionary6: OleVariant);

- procedure TWordDocument.CheckSpelling(var CustomDictionary: OleVariant;var IgnoreUppercase: OleVariant;var AlwaysSuggest: OleVariant;var CustomDictionary2: OleVariant;var CustomDictionary3: OleVariant;var CustomDictionary4: OleVariant;var 
CustomDictionary5: OleVariant;var CustomDictionary6: OleVariant;var CustomDictionary7: OleVariant);

- procedure TWordDocument.CheckSpelling(var CustomDictionary: OleVariant;var IgnoreUppercase: OleVariant;var AlwaysSuggest: OleVariant;var CustomDictionary2: OleVariant;var CustomDictionary3: OleVariant;var CustomDictionary4: OleVariant;var CustomDictionary5: OleVariant;var CustomDictionary6: OleVariant;var CustomDictionary7: OleVariant;var CustomDictionary8: OleVariant);

- procedure TWordDocument.CheckSpelling(var CustomDictionary: OleVariant;var IgnoreUppercase: OleVariant;var AlwaysSuggest: OleVariant;var CustomDictionary2: OleVariant;var CustomDictionary3: OleVariant;var CustomDictionary4: OleVariant;var CustomDictionary5: OleVariant;var CustomDictionary6: OleVariant;var CustomDictionary7: OleVariant;var CustomDictionary8: OleVariant;var CustomDictionary9: OleVariant);

- procedure TWordDocument.FollowHyperlink;

- procedure TWordDocument.FollowHyperlink(var Address: OleVariant);

- procedure TWordDocument.FollowHyperlink(var Address: OleVariant; var SubAddress: OleVariant);var EmptyParam: OleVariant;

- procedure TWordDocument.FollowHyperlink(var Address: OleVariant; var SubAddress: OleVariant; var NewWindow: OleVariant);

- procedure TWordDocument.FollowHyperlink(var Address: OleVariant; var SubAddress: OleVariant; var NewWindow: OleVariant; var AddHistory: OleVariant);

- procedure TWordDocument.FollowHyperlink(var Address: OleVariant; var SubAddress: OleVariant; var NewWindow: OleVariant; var AddHistory: OleVariant; var ExtraInfo: OleVariant);

- procedure TWordDocument.FollowHyperlink(var Address: OleVariant; var SubAddress: OleVariant; var NewWindow: OleVariant; var AddHistory: OleVariant; var ExtraInfo: OleVariant; var Method: OleVariant);

- function TWordDocument.AutoSummarize: WordRange;

- function TWordDocument.AutoSummarize(var Length: OleVariant): WordRange;

- function TWordDocument.AutoSummarize(var Length: OleVariant; var Mode: OleVariant): WordRange;

- procedure TWordDocument.RemoveNumbers;

- procedure TWordDocument.ConvertNumbersToText;

- function TWordDocument.CountNumberedItems: Integer;

- function TWordDocument.CountNumberedItems(var NumberType: OleVariant): Integer;

- procedure TWordDocument.SendMailer;

- procedure TWordDocument.SendMailer(var FileFormat: OleVariant);

- procedure TWordDocument.SendFax(const Address: WideString);

- function TWordDocument.CreateLetterContent(const DateFormat: WideString; IncludeHeaderFooter: WordBool; const PageDesign: WideString; LetterStyle: WdLetterStyle; Letterhead: WordBool; LetterheadLocation: WdLetterheadLocation; LetterheadSize: Single; const RecipientName: WideString; $\quad$ const RecipientAddress: WideString; const Salutation: WideString; SalutationType: WdSalutationType; const RecipientReference: WideString; const MailingInstructions: WideString; const AttentionLine: WideString; const Subject: WideString; const CCList: WideString; const ReturnAddress: WideString; const SenderName: WideString; const Closing: WideString; const SenderCompany: WideString; const SenderJobTitle: WideString; const SenderInitials: WideString; EnclosureNumber: Integer): WordLetterContent;

- function TWordDocument.CreateLetterContent(const DateFormat: WideString; IncludeHeaderFooter: WordBool; const PageDesign: WideString; LetterStyle: 
WdLetterStyle; Letterhead: WordBool; LetterheadLocation: WdLetterheadLocation; LetterheadSize: Single; const RecipientName: WideString; const RecipientAddress: WideString; const Salutation: WideString; SalutationType: WdSalutationType; const RecipientReference: WideString; const MailingInstructions: WideString; const AttentionLine: WideString; const Subject: WideString; const CCList: WideString; const ReturnAddress: WideString; const SenderName: WideString; const Closing: WideString; const SenderCompany: WideString; const SenderJobTitle: WideString; const SenderInitials: WideString; EnclosureNumber: Integer; var InfoBlock: OleVariant): WordLetterContent;

- function TWordDocument.CreateLetterContent(const DateFormat: WideString; IncludeHeaderFooter: WordBool; const PageDesign: WideString; LetterStyle: WdLetterStyle; Letterhead: WordBool; LetterheadLocation: WdLetterheadLocation; LetterheadSize: Single; const RecipientName: WideString; const RecipientAddress: WideString; const Salutation: WideString; SalutationType: WdSalutationType; const RecipientReference: WideString; const MailingInstructions: WideString; const AttentionLine: WideString; const Subject: WideString; const CCList: WideString; const ReturnAddress: WideString; const SenderName: WideString; const Closing: WideString; const SenderCompany: WideString; const SenderJobTitle: WideString; const SenderInitials: WideString; EnclosureNumber: Integer; var InfoBlock: OleVariant; var RecipientCode: OleVariant): WordLetterContent;

- function TWordDocument.CreateLetterContent(const DateFormat: WideString; IncludeHeaderFooter: WordBool; const PageDesign: WideString; LetterStyle: WdLetterStyle; Letterhead: WordBool; LetterheadLocation: WdLetterheadLocation; LetterheadSize: Single; const RecipientName: WideString; const RecipientAddress: WideString; const Salutation: WideString; SalutationType: WdSalutationType; const RecipientReference: WideString; const MailingInstructions: WideString; const AttentionLine: WideString; const Subject: WideString; const CCList: WideString; const ReturnAddress: WideString; const SenderName: WideString; const Closing: WideString; const SenderCompany: WideString; const SenderJobTitle: WideString; const SenderInitials: WideString; EnclosureNumber: Integer; var InfoBlock: OleVariant; var RecipientCode: OleVariant; $\quad$ var RecipientGender: OleVariant): WordLetterContent;

- function TWordDocument.CreateLetterContent(const DateFormat: WideString; IncludeHeaderFooter: WordBool; const PageDesign: WideString; LetterStyle: WdLetterStyle; Letterhead: WordBool; LetterheadLocation: WdLetterheadLocation; LetterheadSize: Single; const RecipientName: WideString; const RecipientAddress: WideString; const Salutation: WideString; SalutationType: WdSalutationType; const RecipientReference: WideString; const MailingInstructions: WideString; const AttentionLine: WideString; const Subject: WideString; const CCList: WideString; const ReturnAddress: WideString; const SenderName: WideString; const Closing: WideString; const SenderCompany: WideString; const SenderJobTitle: WideString; const SenderInitials: WideString; EnclosureNumber: Integer; var InfoBlock: OleVariant; var RecipientCode: OleVariant; var RecipientGender: OleVariant; var ReturnAddressShortForm: OleVariant): WordLetterContent;

- function TWordDocument.CreateLetterContent(const DateFormat: WideString; IncludeHeaderFooter: WordBool; const PageDesign: WideString; LetterStyle: WdLetterStyle; Letterhead: WordBool; LetterheadLocation: WdLetterheadLocation; LetterheadSize: Single; const RecipientName: WideString; const RecipientAddress: WideString; const Salutation: WideString; SalutationType: WdSalutationType; const RecipientReference: WideString; const MailingInstructions: WideString; const AttentionLine: WideString; const Subject: WideString; const CCList: WideString; const ReturnAddress: WideString; const SenderName: WideString; const Closing: 
WideString; const SenderCompany: WideString; const SenderJobTitle: WideString; const SenderInitials: WideString; EnclosureNumber: Integer; var InfoBlock: OleVariant; var RecipientCode: OleVariant; var RecipientGender: OleVariant; var ReturnAddressShortForm: OleVariant; var SenderCity: OleVariant): WordLetterContent;

- function TWordDocument.CreateLetterContent(const DateFormat: WideString; IncludeHeaderFooter: WordBool; const PageDesign: WideString; LetterStyle: WdLetterStyle; Letterhead: WordBool; LetterheadLocation: WdLetterheadLocation; LetterheadSize: Single; const RecipientName: WideString; const RecipientAddress: WideString; const Salutation: WideString; SalutationType: WdSalutationType; const RecipientReference: WideString; const MailingInstructions: WideString; const AttentionLine: WideString; const Subject: WideString; const CCList: WideString; const ReturnAddress: WideString; const SenderName: WideString; const Closing: WideString; const SenderCompany: WideString; const SenderJobTitle: WideString; const SenderInitials: WideString; EnclosureNumber: Integer; var InfoBlock: OleVariant; var RecipientCode: OleVariant; var RecipientGender: OleVariant; var ReturnAddressShortForm: OleVariant; var SenderCity: OleVariant; var SenderCode: OleVariant): WordLetterContent;

- function TWordDocument.CreateLetterContent(const DateFormat: WideString; IncludeHeaderFooter: WordBool; const PageDesign: WideString; LetterStyle: WdLetterStyle; Letterhead: WordBool; LetterheadLocation: WdLetterheadLocation; LetterheadSize: Single; const RecipientName: WideString; const RecipientAddress: WideString; const Salutation: WideString; SalutationType: WdSalutationType; const RecipientReference: WideString; const MailingInstructions: WideString; const AttentionLine: WideString; const Subject: WideString; const CCList: WideString; const ReturnAddress: WideString; const SenderName: WideString; const Closing: WideString; const SenderCompany: WideString; const SenderJobTitle: WideString; const SenderInitials: WideString; $\quad$ EnclosureNumber: Integer; var InfoBlock: OleVariant; var RecipientCode: OleVariant; var RecipientGender: OleVariant; var ReturnAddressShortForm: OleVariant; var SenderCity: OleVariant; var SenderCode: OleVariant; var SenderGender: OleVariant): WordLetterContent;

- procedure TWordDocument.PrintOut2000;

- procedure TWordDocument.PrintOut2000(var Background: OleVariant);

- procedure TWordDocument.PrintOut2000(var Background: OleVariant; var Append: OleVariant);

- procedure TWordDocument.PrintOut2000(var Background: OleVariant; var Append: OleVariant; $\quad$ var Range: OleVariant);

- procedure TWordDocument.PrintOut2000(var Background: OleVariant; var Append: OleVariant; $\quad$ var Range: OleVariant; var OutputFileName: OleVariant);

- procedure TWordDocument.PrintOut2000(var Background: OleVariant; var Append: OleVariant; $\quad$ var Range: OleVariant; var OutputFileName: OleVariant; $\quad$ var From: OleVariant);

- procedure TWordDocument.PrintOut2000(var Background: OleVariant; OleVariant; $\quad$ var Range: OleVariant; var OutputFileName: OleVariant;

var Append: OleVariant; var To_: OleVariant);

- procedure TWordDocument.PrintOut2000(var Background: OleVariant; var Append: OleVariant; $\quad$ var Range: OleVariant; var OutputFileName: OleVariant; $\quad$ var From: OleVariant; var To_: OleVariant; var Item: OleVariant); 
- procedure TWordDocument.PrintOut2000(var Background: OleVariant; var Append: OleVariant; $\quad$ var Range: OleVariant; var OutputFileName: OleVariant; var From: OleVariant; var To_: OleVariant; $\quad$ var Item: OleVariant; var Copies: OleVariant);

- procedure TWordDocument.PrintOut2000(var Background: OleVariant; var Append: OleVariant; $\quad$ var Range: OleVariant; var OutputFileName: OleVariant; var From: OleVariant; var To_: OleVariant; $\quad$ var Item: OleVariant; var Copies: OleVariant; var Pages: OleVariant);

- procedure TWordDocument.PrintOut2000(var Background: OleVariant; var Append: OleVariant; $\quad$ var Range: OleVariant; var OutputFileName: OleVariant; var From: OleVariant; var To_: OleVariant; $\quad$ var Item: OleVariant; var Copies: OleVariant; var Pages: OleVariant; var PageType: OleVariant);

- procedure TWordDocument.PrintOut2000(var Background: OleVariant; var Append: OleVariant; $\quad$ var Range: OleVariant; var OutputFileName: OleVariant; var From: OleVariant; var To_: OleVariant; $\quad$ var Item: OleVariant; var Copies: OleVariant; var Pages: OleVariant; var PageType: OleVariant; $\quad$ var PrintToFile: OleVariant);

- procedure TWordDocument.PrintOut2000(var Background: OleVariant; var Append: OleVariant; $\quad$ var Range: OleVariant; var OutputFileName: OleVariant; var From: OleVariant; var To_: OleVariant; var Item: OleVariant; var Copies: OleVariant; var Pages: OleVariant; var PageType: OleVariant; $\quad$ var PrintToFile: OleVariant; var Collate: OleVariant);

- procedure TWordDocument.PrintOut2000(var Background: OleVariant; var Append: OleVariant; $\quad$ var Range: OleVariant; var OutputFileName: OleVariant; var From: OleVariant; var To_: OleVariant; $\quad$ var Item: OleVariant; var Copies: OleVariant; var Pages: OleVariant; var PageType: OleVariant; $\quad$ var PrintToFile: OleVariant; var Collate: OleVariant; $\quad$ var ActivePrinterMacGX: OleVariant);

- procedure TWordDocument.PrintOut2000(var Background: OleVariant; var Append: OleVariant; $\quad$ var Range: OleVariant; var OutputFileName: OleVariant; $\quad$ var From: OleVariant; var To_: OleVariant; var Item: OleVariant; var Copies: OleVariant; var Pages: OleVariant; var PageType: OleVariant; $\quad$ var PrintToFile: OleVariant; var Collate: OleVariant; var ActivePrinterMacGX: OleVariant; var ManualDuplexPrint: OleVariant);

- procedure TWordDocument.PrintOut2000(var Background: OleVariant; var Append: OleVariant; $\quad$ var Range: OleVariant; var OutputFileName: OleVariant; var From: OleVariant; var To_: OleVariant; var Item: OleVariant; var Copies: OleVariant; var Pages: OleVariant; var PageType: OleVariant; $\quad$ var PrintToFile: OleVariant; var Collate: OleVariant; var ActivePrinterMacGX: OleVariant; var ManualDuplexPrint: OleVariant; $\quad$ var PrintZoomColumn: OleVariant);

- procedure TWordDocument.PrintOut2000(var Background: OleVariant; var Append: OleVariant; $\quad$ var Range: OleVariant; var OutputFileName: OleVariant; var From: OleVariant; var To_: OleVariant; var Item: OleVariant; var Copies: OleVariant; var Pages: OleVariant; var PageType: OleVariant; $\quad$ var PrintToFile: OleVariant; var Collate: OleVariant; var ActivePrinterMacGX: OleVariant; var ManualDuplexPrint: OleVariant; var PrintZoomColumn: OleVariant; var PrintZoomRow: OleVariant);

- procedure TWordDocument.PrintOut2000(var Background: OleVariant; var Append: OleVariant; $\quad$ var Range: OleVariant; var OutputFileName: OleVariant; var From: OleVariant; var To_: OleVariant; $\quad$ var Item: OleVariant; var Copies: OleVariant; var Pages: OleVariant; var PageType: OleVariant; var PrintToFile: OleVariant; var Collate: OleVariant; var ActivePrinterMacGX: OleVariant; var 
ManualDuplexPrint: OleVariant;

var PrintZoomColumn: OleVariant; var PrintZoomRow: OleVariant; $\quad$ var PrintZoomPaperWidth: OleVariant);

- procedure TWordDocument.PrintOut;

- procedure TWordDocument.PrintOut(var Background: OleVariant);

- procedure TWordDocument.PrintOut(var Background: OleVariant; var Append: OleVariant);

- procedure TWordDocument.PrintOut(var Background: OleVariant; var Append: OleVariant; var Range: OleVariant);

- procedure TWordDocument.PrintOut(var Background: OleVariant; var Append: OleVariant; var Range: OleVariant; var OutputFileName: OleVariant);

- procedure TWordDocument.PrintOut(var Background: OleVariant; var Append: OleVariant; var Range: OleVariant; var OutputFileName: OleVariant; var From: OleVariant);

- procedure TWordDocument.PrintOut(var Background: OleVariant; var Append: OleVariant; $\quad$ var Range: OleVariant; var OutputFileName: OleVariant; $\quad$ var From: OleVariant; var To_: OleVariant);

- procedure TWordDocument.PrintOut(var Background: OleVariant; var Append: OleVariant; $\quad$ var Range: OleVariant; var OutputFileName: OleVariant; $\quad$ var From: OleVariant; var To_: OleVariant; var Item: OleVariant);

- procedure TWordDocument.PrintOut(var Background: OleVariant; var Append: OleVariant; var Range: OleVariant; var OutputFileName: OleVariant; var From: OleVariant; var To_: OleVariant; var Item: OleVariant; var Copies: OleVariant);

- procedure TWordDocument.PrintOut(var Background: OleVariant; var Append: OleVariant; $\quad$ var Range: OleVariant; var OutputFileName: OleVariant; var From: OleVariant; var To_: OleVariant; var Item: OleVariant; var Copies: OleVariant; var Pages: OleVariant);

- procedure TWordDocument.PrintOut(var Background: OleVariant; var Append: OleVariant; var Range: OleVariant; var OutputFileName: OleVariant; var From: OleVariant; var To_: OleVariant; var Item: OleVariant; var Copies: OleVariant; var Pages: OleVariant; $\quad$ var PageType: OleVariant);

- procedure TWordDocument.PrintOut(var Background: OleVariant; var Append: OleVariant; var Range: OleVariant; var OutputFileName: OleVariant; var From: OleVariant; var To_: OleVariant; var Item: OleVariant; var Copies: OleVariant; var Pages: OleVariant; $\quad$ var PageType: OleVariant; var PrintToFile: OleVariant);

- procedure TWordDocument.PrintOut(var Background: OleVariant; var Append: OleVariant; var Range: OleVariant; var OutputFileName: OleVariant; var From: OleVariant; var To_: OleVariant; var Item: OleVariant; var Copies: OleVariant; var Pages: OleVariant; $\quad$ var PageType: OleVariant; var PrintToFile: OleVariant; var Collate: OleVariant);

- procedure TWordDocument.PrintOut(var Background: OleVariant; var Append: OleVariant; var Range: OleVariant; var OutputFileName: OleVariant; var From: OleVariant; var To_: OleVariant; var Item: OleVariant; var Copies: OleVariant; var Pages: OleVariant; $\quad$ var PageType: OleVariant; var PrintToFile: OleVariant; var Collate: OleVariant; var ActivePrinterMacGX: OleVariant);

- procedure TWordDocument.PrintOut(var Background: OleVariant; var Append: OleVariant; var Range: OleVariant; var OutputFileName: OleVariant; var From: OleVariant; var To_: OleVariant; var Item: OleVariant; var Copies: OleVariant; var Pages: OleVariant; var PageType: OleVariant; var PrintToFile: OleVariant; var Collate: OleVariant; var ActivePrinterMacGX: OleVariant; $\quad$ var ManualDuplexPrint: OleVariant); 
- procedure TWordDocument.PrintOut(var Background: OleVariant; var Append: OleVariant; var Range: OleVariant; var OutputFileName: OleVariant; var From: OleVariant; var To_: OleVariant; var Item: OleVariant; var Copies: OleVariant; var Pages: OleVariant; var PageType: OleVariant; var PrintToFile: OleVariant; var Collate: OleVariant; var ActivePrinterMacGX: OleVariant; var ManualDuplexPrint: OleVariant; var PrintZoomColumn: OleVariant);

- procedure TWordDocument.PrintOut(var Background: OleVariant; var Append: OleVariant; var Range: OleVariant; var OutputFileName: OleVariant; var From: OleVariant; var To_: OleVariant; var Item: OleVariant; var Copies: OleVariant; var Pages: OleVariant; var PageType: OleVariant; var PrintToFile: OleVariant; var Collate: OleVariant; var ActivePrinterMacGX: OleVariant; var ManualDuplexPrint: OleVariant; var PrintZoomColumn: OleVariant; var PrintZoomRow: OleVariant);

- procedure TWordDocument.PrintOut(var Background: OleVariant; var Append: OleVariant; var Range: OleVariant; var OutputFileName: OleVariant; var From: OleVariant; var To_: OleVariant; var Item: OleVariant; var Copies: OleVariant; var Pages: OleVariant; var PageType: OleVariant; var PrintToFile: OleVariant; var Collate: OleVariant; var ActivePrinterMacGX: OleVariant; var ManualDuplexPrint: OleVariant; var PrintZoomColumn: OleVariant; var PrintZoomRow: OleVariant; var PrintZoomPaperWidth: OleVariant);

- procedure TWordDocument.Compare2002(const Name: WideString);

- procedure TWordDocument.Compare2002(const Name: WideString; var AuthorName: OleVariant);

- procedure TWordDocument.Compare2002(const Name: WideString; var AuthorName: OleVariant; $\quad$ var CompareTarget: OleVariant);

- procedure TWordDocument.Compare2002(const Name: WideString; var AuthorName: OleVariant; var CompareTarget: OleVariant; var DetectFormatChanges: OleVariant);

- procedure TWordDocument.Compare2002(const Name: WideString; var AuthorName: OleVariant; var CompareTarget: OleVariant; var DetectFormatChanges: OleVariant; $\quad$ var IgnoreAllComparisonWarnings: OleVariant);

- procedure TWordDocument.Merge(const FileName: WideString);

- procedure TWordDocument.Merge(const FileName: WideString; var MergeTarget: OleVariant);

- procedure TWordDocument.Merge(const FileName: WideString; var MergeTarget: OleVariant;

- procedure TWordDocument.Merge(const FileName: WideString; var MergeTarget: OleVariant; var DetectFormatChanges: OleVariant);

- procedure TWordDocument.Merge(const FileName: WideString; var MergeTarget: OleVariant; var DetectFormatChanges: OleVariant; var UseFormattingFrom: OleVariant);

- procedure TWordDocument.SendForReview;

- procedure TWordDocument.SendForReview(var Recipients: OleVariant);

- procedure TWordDocument.SendForReview(var Recipients: OleVariant; var Subject: OleVariant);

- procedure TWordDocument.SendForReview(var Recipients: OleVariant; var Subject: OleVariant;var ShowMessage: OleVariant);

- procedure TWordDocument.ReplyWithChanges;

- procedure TWordDocument.SetPasswordEncryptionOptions(const PasswordEncryptionProvider: WideString; const PasswordEncryptionAlgorithm: WideString; PasswordEncryptionKeyLength: Integer);

- procedure TWordDocument.SaveAs; 
- procedure TWordDocument.SaveAs(var FileName: OleVariant);

- procedure TWordDocument.SaveAs(var FileName: OleVariant; var FileFormat: OleVariant);

- procedure TWordDocument.SaveAs(var FileName: OleVariant; var FileFormat: OleVariant; var LockComments: OleVariant);

- procedure TWordDocument.SaveAs(var FileName: OleVariant; var FileFormat: OleVariant; var LockComments: OleVariant; var Password: OleVariant);

- procedure TWordDocument.SaveAs(var FileName: OleVariant; var FileFormat: OleVariant; var LockComments: OleVariant; var Password: OleVariant; var AddToRecentFiles: OleVariant);

- procedure TWordDocument.SaveAs(var FileName: OleVariant; var FileFormat: OleVariant; var LockComments: OleVariant; var Password: OleVariant; var AddToRecentFiles: OleVariant; var WritePassword: OleVariant);

- procedure TWordDocument.SaveAs(var FileName: OleVariant; var FileFormat: OleVariant; var LockComments: OleVariant; var Password: OleVariant; var AddToRecentFiles: OleVariant; var WritePassword: OleVariant; var ReadOnlyRecommended: OleVariant);

- procedure TWordDocument.SaveAs(var FileName: OleVariant; var FileFormat: OleVariant; var LockComments: OleVariant; var Password: OleVariant; var AddToRecentFiles: OleVariant; var WritePassword: OleVariant; var ReadOnlyRecommended: OleVariant; var EmbedTrueTypeFonts: OleVariant);

- procedure TWordDocument.SaveAs(var FileName: OleVariant; var FileFormat: OleVariant; var LockComments: OleVariant; var Password: OleVariant; var AddToRecentFiles: OleVariant; var WritePassword: OleVariant; var ReadOnlyRecommended: OleVariant; var EmbedTrueTypeFonts: OleVariant; var SaveNativePictureFormat: OleVariant);

- procedure TWordDocument.SaveAs(var FileName: OleVariant; var FileFormat: OleVariant; var LockComments: OleVariant; var Password: OleVariant; var AddToRecentFiles: OleVariant; var WritePassword: OleVariant; var ReadOnlyRecommended: OleVariant; var EmbedTrueTypeFonts: OleVariant; var SaveNativePictureFormat: OleVariant; var SaveFormsData: OleVariant);

- procedure TWordDocument.SaveAs(var FileName: OleVariant; var FileFormat: OleVariant; var LockComments: OleVariant; var Password: OleVariant; var AddToRecentFiles: OleVariant; var WritePassword: OleVariant; var ReadOnlyRecommended: OleVariant; var EmbedTrueTypeFonts: OleVariant; var SaveNativePictureFormat: OleVariant; var SaveFormsData: OleVariant; var SaveAsAOCELetter: OleVariant);

- procedure TWordDocument.SaveAs(var FileName: OleVariant; var FileFormat: OleVariant; var LockComments: OleVariant; var Password: OleVariant; var AddToRecentFiles: OleVariant; var WritePassword: OleVariant; var ReadOnlyRecommended: OleVariant; var EmbedTrueTypeFonts: OleVariant; var SaveNativePictureFormat: OleVariant; var SaveFormsData: OleVariant; var SaveAsAOCELetter: OleVariant; var Encoding: OleVariant);

- procedure TWordDocument.SaveAs(var FileName: OleVariant; var FileFormat: OleVariant; var LockComments: OleVariant; var Password: OleVariant; var AddToRecentFiles: OleVariant; var WritePassword: OleVariant; var ReadOnlyRecommended: OleVariant; var EmbedTrueTypeFonts: OleVariant; var SaveNativePictureFormat: OleVariant; var SaveFormsData: OleVariant; var SaveAsAOCELetter: OleVariant; var Encoding: OleVariant; var InsertLineBreaks: OleVariant); 
- procedure TWordDocument.SaveAs(var FileName: OleVariant; var FileFormat: OleVariant; var LockComments: OleVariant; var Password: OleVariant; var AddToRecentFiles: OleVariant; var WritePassword: OleVariant; var ReadOnlyRecommended: OleVariant; var EmbedTrueTypeFonts: OleVariant; var SaveNativePictureFormat: OleVariant; var SaveFormsData: OleVariant; var SaveAsAOCELetter: OleVariant; var Encoding: OleVariant; var InsertLineBreaks: OleVariant; var AllowSubstitutions: OleVariant);

- procedure TWordDocument.SaveAs(var FileName: OleVariant; var FileFormat: OleVariant; var LockComments: OleVariant; var Password: OleVariant; var AddToRecentFiles: OleVariant; var WritePassword: OleVariant; var ReadOnlyRecommended: OleVariant; var EmbedTrueTypeFonts: OleVariant; var SaveNativePictureFormat: OleVariant; var SaveFormsData: OleVariant; var SaveAsAOCELetter: OleVariant; var Encoding: OleVariant; var InsertLineBreaks: OleVariant; var AllowSubstitutions: OleVariant; var LineEnding: OleVariant);

- procedure TWordDocument.SaveAs(var FileName: OleVariant; var FileFormat: OleVariant; var LockComments: OleVariant; var Password: OleVariant; var AddToRecentFiles: OleVariant; var WritePassword: OleVariant; var ReadOnlyRecommended: OleVariant; var EmbedTrueTypeFonts: OleVariant; var SaveNativePictureFormat: OleVariant; var SaveFormsData: OleVariant; var SaveAsAOCELetter: procedure TWordDocument.SendFaxOverInternet;

- procedure TWordDocument.SendFaxOverInternet(var Recipients: OleVariant);

- procedure TWordDocument.SendFaxOverInternet(var Recipients: OleVariant; var Subject: OleVariant);

- procedure TWordDocument.Protect(Type_: WdProtectionType);

- procedure TWordDocument.Protect(Type_: WdProtectionType; var NoReset: OleVariant);

- procedure TWordDocument.Protect(Type_: WdProtectionType; var NoReset: OleVariant; var Password: OleVariant);

- procedure TWordDocument.Protect(Type_: WdProtectionType; var NoReset: OleVariant; var Password: OleVariant; var UseIRM: OleVariant);

- procedure TWordDocument.SelectAllEditableRanges;

- procedure TWordDocument.DeleteAllEditableRanges;

- procedure TWordDocument.Compare(const Name: WideString);

- procedure TWordDocument.Compare(const Name: WideString; var AuthorName: OleVariant);

- procedure TWordDocument.Compare(const Name: WideString; var AuthorName: OleVariant; var CompareTarget: OleVariant);

- procedure TWordDocument.Compare(const Name: WideString; var AuthorName: OleVariant; var CompareTarget: OleVariant; var DetectFormatChanges: OleVariant);

- procedure TWordDocument.Compare(const Name: WideString; var AuthorName: OleVariant; var CompareTarget: OleVariant; var DetectFormatChanges: OleVariant; var IgnoreAllComparisonWarnings: OleVariant);

- procedure TWordDocument.Compare(const Name: WideString; var AuthorName: OleVariant; var CompareTarget: OleVariant; var DetectFormatChanges: OleVariant; var IgnoreAllComparisonWarnings: OleVariant; var AddToRecentFiles: OleVariant);

- procedure TWordDocument.Compare(const Name: WideString; var AuthorName: OleVariant; var CompareTarget: OleVariant; var DetectFormatChanges: OleVariant; var IgnoreAllComparisonWarnings: OleVariant; var AddToRecentFiles: OleVariant; var RemovePersonalInformation: OleVariant);

- procedure TWordDocument.ExportAsFixedFormat(const OutputFileName: WideString; ExportFormat: WdExportFormat; OpenAfterExport: WordBool; OptimizeFor: 
WdExportOptimizeFor; Range: WdExportRange;

WdExportItem; IncludeDocProps:

CreateBookmarks: WdExportCreateBookmarks;

BitmapMissingFonts: WordBool; UseISO19005_1: WordBool);
From: Integer; To_: Integer; Item: WordBool; KeepIRM: WordBool; DocStructureTags: WordBool;

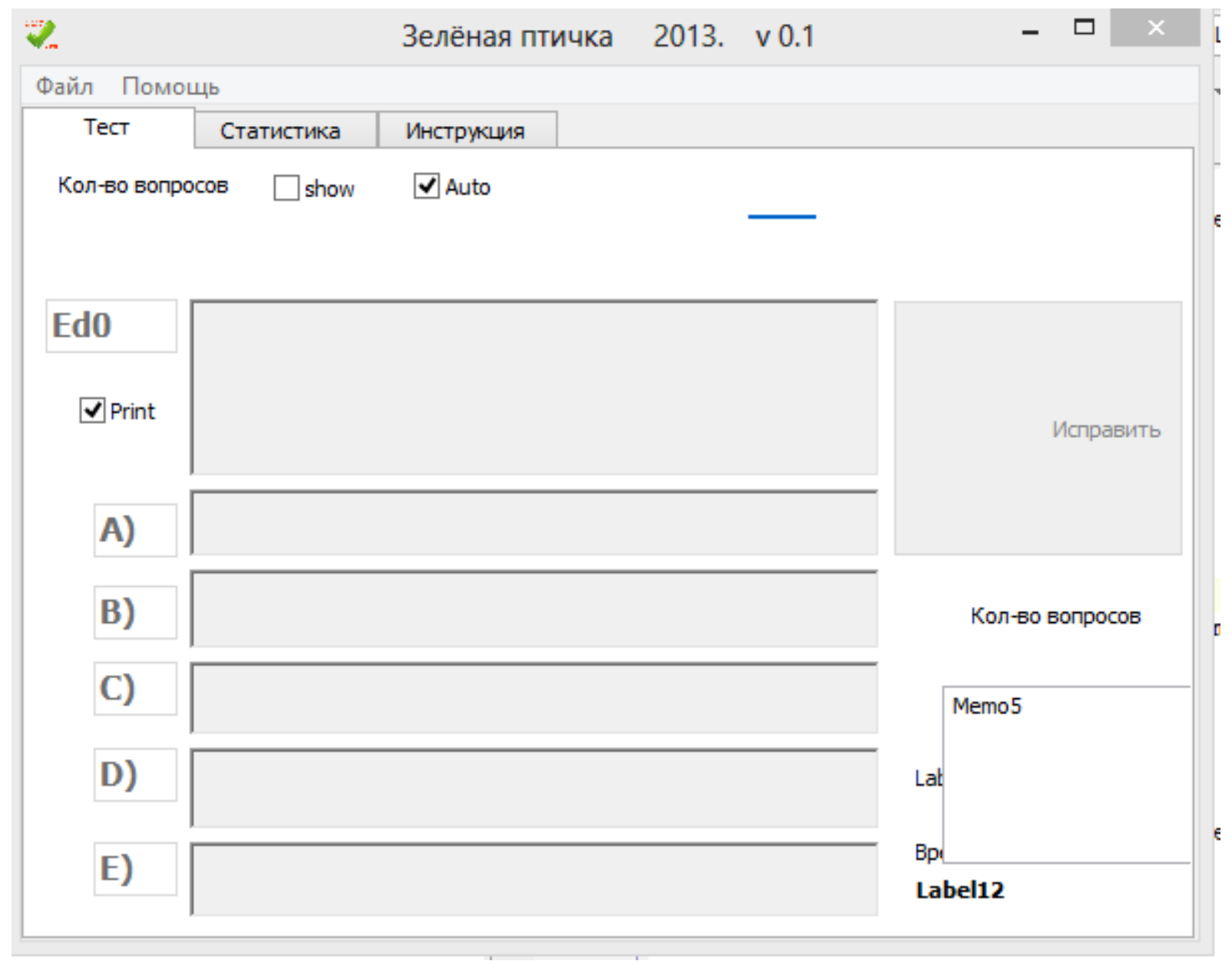

Рисунок 2 - Отсутствие ошибок компиляции Word_TLB.dcu.

Следует отметить, что при отключении Word_TLB.dcu в разделе Uses, программа работает и без этой библиотеки, но при этом придется выключить процедуру смещения:

WordApp1.Selection.EndKey(wdStory,EmptyParam);

Также была замечена ошибка выхода из таблицы при подаче команды (1). Смещение курсора не происходило, вместо этого он оставался в клетке $[1,1]$ таблицы.

\section{References:}

1. Шевцов А.Н., Шырынханова Д.Ж. Разработка алгоритмов и приложения компонентной модели для анализа и исправления ошибок экзаменационного теста. Theoretical \& Applied Science. «Development of Applied Mathematics», ISPC, 30.05.2013, Taraz, Kazakhstan. - №5, 2013. -p.77-83. [Электронный ресурс] URL: http://elibrary.ru/item.asp?id=20357857 (дата доступа 28.04.2014) 
2. Шевцов А.Н., Смайлова У.М., Шырынханова Д.Ж. Некоторые алгоритмы предварительной обработки теста. Theoretical \& Applied Science. «Results \& Perspectives», ISPC, 30.09.2013, Florence, Italy. - №9, 2013. -p.51-58. [Электронный pecypc] URL: http://elibrary.ru/item.asp?id=20362250 (дата доступа 28.04.2014)

3. Ошибка при компиляции: Could not create output file 'Unit2.dcu' / Delphi для начинающих [Электронный ресурc] URL: http://www.cyberforum.ru/delphibeginners/thread862975.html (дата доступа 16.04.2014)

4. Delphi и OLE Automation c Word [Электронный pecypc] URL: http://delphiworld.narod.ru/base/delphi_and_word_ole_automation.html (дата доступа 16.04.2014)

5. Тенцер А. Создание модулей расширения Microsoft Office [Электронный ресурс] URL: http://www.infocity.kiev.ua/prog/delphi/content/delphi087.phtml (дата доступа 16.04.2014)

6. Советы по Delphi. Версия 1.4.3 от 1.1.2001 (fb2) [Электронный ресурc] URL: http://coollib.com/b/121413/read (дата доступа 16.04.2014)

7. 500+ FAQ по Delphi [Электронный pecypc] URL: http://develab.narod.ru/delphi_faq/01.htm (дата доступа 16.04.2014)

8. Управление приложениями пакета MS Office. Управление MS Word.

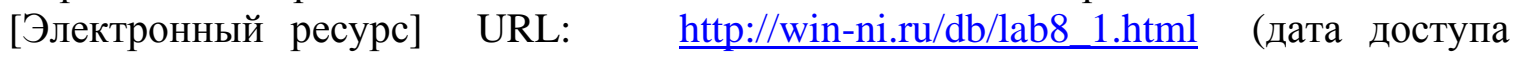
16.04.2014)

9. Тестирование [Электронный pecypc] URL: http://www.helloworld.ru/texts/comp/lang/delphi/delphi4/test.htm _ дата доступа 28.04.2014)

10. Уроки Delphi / Система проверки знаний. (оболочка теста на Delphi) [Электронный ресурc] URL: http://delphicomponent.ru/131-uroki-delphi-sistemaproverki-znaniy-obolochka-testa-na-delphi.html (дата доступа 28.04.2014) 